\title{
Correction: A methodological survey of the analysis, reporting and interpretation of Absolute Risk Reduction in systematic revieWs (ARROW): a study protocol
}

\author{
Pablo Alonso-Coello ${ }^{1,2^{*}}$, Alonso Carrasco-Labra ${ }^{2,3}$, Romina Brignardello-Petersen ${ }^{2,3}$, Ignacio Neumann ${ }^{2,4}$, Elie A Ak| $\left.\right|^{2,5,6}$, \\ Xin Sun ${ }^{2,7}$, Bradley C Johnston ${ }^{2,8,9}$, Matthias Briel ${ }^{10}$, Jason W Busse ${ }^{2,11}$, Demián Glujovsky ${ }^{12}$, Carlos E Granados ${ }^{13}$, \\ Alfonso lorio ${ }^{2}$, Affan Irfan ${ }^{14}$, Laura Martínez García', Reem A Mustafa ${ }^{15}$, Anggie Ramirez-Morera ${ }^{16}$, Iván Solà', \\ Kari A O Tikkinen 2,17, Shanil Ebrahim²,11,18, Per O Vandvik ${ }^{19}$, Yuqing Zhang ${ }^{2}$, Anna Selva', Andrea J Sanabria', \\ Oscar E Zazueta', Robin W M Vernooij ${ }^{1}$, Holger J Schünemann² and Gordon H Guyatt ${ }^{2}$
}

\section{Correction}

Following publication of our article [1], it has come to our attention that Laura Martínez García's surname was displayed incorrectly. We publish this correction to update the author list, which is as follows:

Pablo Alonso-Coello, Alonso Carrasco-Labra, Romina Brignardello-Petersen, Ignacio Neumann, Elie A Akl, Xin Sun, Bradley C Johnston, Matthias Briel, Jason W Busse, Demián Glujovsky, Carlos E Granados, Alfonso Iorio, Affan Irfan, Laura Martínez García, Reem A Mustafa, Anggie Ramirez-Morera, Iván Solà, Kari A O Tikkinen, Shanil Ebrahim, Per O Vandvik, Yuqing Zhang, Anna Selva, Andrea J Sanabria, Oscar E Zazueta, Robin W M Vernooij, Holger J Schünemann and Gordon H Guyatt.

\footnotetext{
Author details

'Iberoamerican Cochrane Centre, Institute of Biomedical Research (IIB Sant Pau), Barcelona, Spain. ${ }^{2}$ Department of Clinical Epidemiology \& Biostatistics, McMaster University, Hamilton, Canada. ${ }^{3}$ Evidence-Based Dentistry Unit, Faculty of Dentistry, Universidad de Chile, Santiago, Chile. ${ }^{4}$ Department of Medicine, Pontificia Universidad Católica de Chile, Santiago, Chile. ${ }^{5}$ Department of Internal Medicine, American University of Beirut, Beirut, Lebanon. ${ }^{6}$ Department of Medicine, State University of New York, Buffalo, NY, USA. ${ }^{7}$ Chinese Evidence-based Medicine Centre, West China Hospital, Sichuan University, Chengdu, China. ${ }^{8}$ Department of Anaesthesia and Pain Medicine, The Hospital for Sick Children, Institute of Health Policy, Management and Evaluation, University of Toronto, Toronto, Ontario, Canada. ${ }^{9} \mathrm{C}$ ild Health Evaluative Sciences, The Hospital for Sick Children Research Institute, Toronto,
}

Ontario, Canada. ${ }^{10}$ Basel Institute for Clinical Epidemiology and Biostatistics, University Hospital Basel, Basel, Switzerland. "'Department of Anaesthesia, McMaster University, Hamilton, Canada. ${ }^{12}$ Argentine Cochrane Centre IECS (Institute for Clinical Effectiveness and Health Policy), Buenos Aires, Argentina. ${ }^{13}$ Área de investigaciones, Facultad de Medicina, Universidad de La Sabana, Chía, Colombia. ${ }^{14}$ Internal Medicine Residency Program, University of Illinois, Urbana-Champaign, Illinois, USA. ${ }^{15}$ Department of Medicine and Nephrology, University Of Missouri-Kansas City, Missouri-Kansas City, Missouri, USA. ${ }^{16} \mathrm{CCSS}$ Permanent Medical Advisor, Health Care Development Division, IHCAI Foundation \& Central America Cochrane, Costa Rica, Costa Rica. ${ }^{17}$ Department of Urology, Helsinki University Central Hospital and University of Helsinki, Helsinki, Finland. ${ }^{18}$ Institute of Health Policy, Management \& Evaluation, University of Toronto, Toronto, Canada. ${ }^{19}$ Norwegian Knowledge Centre for the Health Services, Oslo, Norway.

Received: 27 June 2014 Accepted: 30 June 2014

Published: 17 July 2014

\section{Reference}

1. Alonso-Coello P, Carrasco-Labra A, Brignardello-Petersen R, Neumann I, Akl EA, Sun X, Johnston BC, Briel M, Busse JW, Glujovsky D, Granados CE, lorio A, Irfan A, García LM, Mustafa RA, Ramirez-Morera A, Solà I, Tikkinen KA, Ebrahim S, Vandvik PO, Zhang Y, Selva A, Sanabria AJ, Zazueta OE, Vernooij RW, Schünemann HJ, Guyatt GH: A methodological survey of the analysis, reporting and interpretation of Absolute Risk ReductiOn in systematic revieWs (ARROW): a study protocol. Syst Rev 2013, 2:113.

doi:10.1186/2046-4053-3-76

Cite this article as: Alonso-Coello et al:: Correction: A methodological survey of the analysis, reporting and interpretation of Absolute Risk Reduction in systematic revieWs (ARROW): a study protocol. Systematic Reviews 2014 3:76.

\footnotetext{
* Correspondence: palonso@santpau.cat

'Iberoamerican Cochrane Centre, Institute of Biomedical Research (IIB Sant Pau), Barcelona, Spain

${ }^{2}$ Department of Clinical Epidemiology \& Biostatistics, McMaster University, Hamilton, Canada
} 